\title{
Primary Bilateral Thalamic Astrocytoma Presenting With Head Tremor, Ataxia, and Dementia
}

\section{To the Editor: Primary bilateral tha- lamic gliomas are exceedingly rare and usually present with behavioral and cognitive abnormalities, sparing motor and sensory functions. ${ }^{1}$ Brain imaging reveals bilateral homoge- neous enlargement of thalamic nuclei, which appear hyperintense on T2-weighted imaging. ${ }^{1}$}

\section{Case Report}

A 42-year-old woman was seen for 6-month progressive behavioral disturbances. At that time she started being unusually aggressive and impatient, complaining of headaches, aching pain in the right leg, and head tremor. The behavioral changes worsened slowly, and she grew incapable of doing things she previously did, such as cooking, cleaning the house, administering medicine to her mother, and caring for her 13-yearold daughter. Relatives reported that she walked with poor balance. When seen, she thought she was in 1970 and in another hospital and that the reason for the consultation was a bloody nipple discharge. Ocular movements were uncoordinated, and she had a rhythmic nodding head tremor, choreoathetoid movement of the right hand, and bilateral cerebellar signs. Muscle strength and sensory functions, as well as visual fields, were normal. Neuropsychological assessment showed moderate to severe impairment of executive function, language, semantic and episodic memory, and visuospatial abilities. Noticeable was a severe

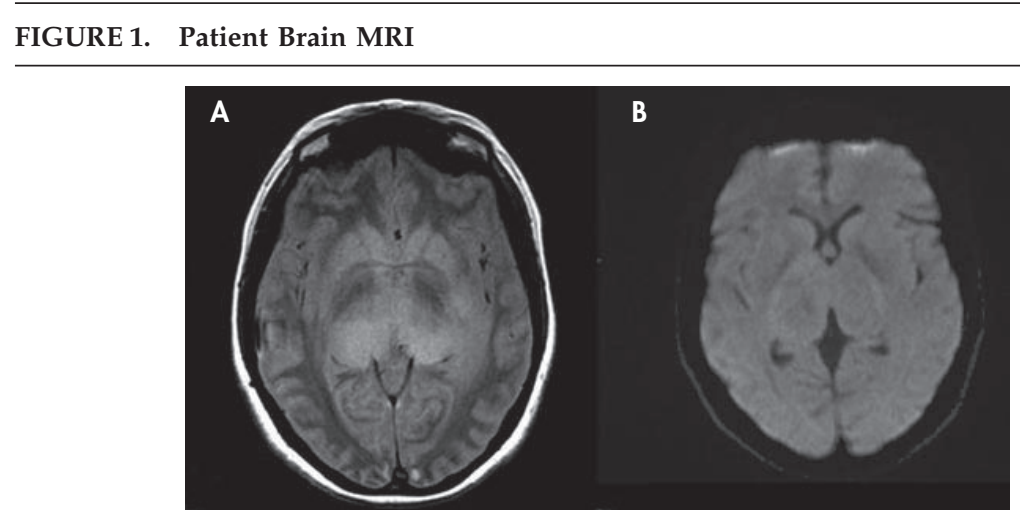

A. PD-weighted axial image shows a hyperintense infiltrative lesion involving both thalami, including the pulvinar; B. DWI reveals slight high signal in the enlarged bilateral thalami (without restricted diffusion)

involvement of temporoparietal cortical functions, with Gerstmann and Balint syndromes.

Patient brain MRI (Figure 1, panels $\mathrm{A}$ and $\mathrm{B}$ ) disclosed a bithalamic diffuse homogeneous lesion, hyperintense on $\mathrm{T} 2$ and iso-intense on T1-weighted images, without restricted diffusivity or gadolinium uptake. EEG showed an 8-Hz background activity. Electromyography characterized the head tremor as rhythmic at $3 \mathrm{~Hz}$. A stereotaxic biopsy was performed, with removal of tissue from the right thalamus.

Histological examination showed astrocytosis with minor nuclear anaplasia and inconspicuous cytoplasm. A few normal large thalamic neurons were found. Spongiform changes were absent, as were mitotic figures. Numerous GFAPpositive processes were present. The diagnosis was of astrocytoma, grade 2 (WHO). On an oncology consultation, only palliative care was proposed. The patient died 3 months later.

\section{Discussion}

As a complex integrating system, the thalami play a central role in ner- vous system function. Pain and dysesthesia can reflect ventral posterior nuclei involvement, since they receive temperature and pain afferents from the contralateral hemibody. ${ }^{2}$ A variety of movement disorders have been related to lesions affecting the ventral inferior or ventral intermediate nuclei, as well as the centromedianum and pulvinar, presumably because of their interconnections with the basal ganglia. ${ }^{3}$ Cerebellar symptoms can result from dysfunction of the ventral anterior, ventral lateral, intralaminar, or reticular nuclei, which are functionally linked to the cerebellum. ${ }^{4}$ The thalami also participate in cortical functioning, by three major networks: temporoparietoccipital, connected with the pulvinar; prefrontal, mainly related with the dorsomedial nuclei; and limbic, which receives efferents from the anterior thalamic nuclei. ${ }^{4}$ Additionally, intralaminar nuclei have a central role in arousal and, with the dorsomedial nuclei, in attention. ${ }^{5}$ We believe these known functions can explain all of our patient's symptoms, even the most atypical and, to our knowledge, not previously described, as the head 


\section{LETTERS}

tremor, the ataxia, and the combined Gerstmann and Balint syndromes.

Álvaro Machado, M.D.

Neurology Department, Hospi-

tal de São Marcos, Braga, Por-

tugal

Manuel Ribeiro, M.D.

Neuroradiology Department,

Hospital de São Marcos

Margarida Rodrigues, M.D.

Carla Ferreira, M.D.

Neurology Department, Hospital de São Marcos

Rui Almeida, M.D.

Neurosurgery Department,
Hospital de São Marcos

Isabel Santana, M.D., Ph.D. Neurology Department, Hospitais da Universidade de Coimbra, Coimbra, Portugal

Lígia Castro, M.D.

Stirling CARPEnTER, M.D. Pathology Department, Hospital de São João, Porto, Portugal

\section{References}

1. Partlow GD, del Carpio-O'Donovan R, Melanson D, et al: Bilateral thalamic glioma: review of eight cases with personality change and mental deterioration. AJNR Am J Neuroradiol 1992; 13:1225-1230

2. Afifi AK, Bergman RA: Functional Neuroanatomy: Text and Atlas. New York, McGraw-Hill, 1998

3. Lee MS, Kim YD, Yang JW, et al: Clinical and anatomical factors associated with thalamic dyskinesias. J Neurol Sci 2001; 182:137-142

4. Perea-Bartolomé MV, Ladera-Fernández V: El tálamo: aspectos neurofuncionales. Rev Neurol 2004; 38:687-693

5. Steriade M, McCormick DA, Sejnowski TJ: Thalamocortical oscillations in the sleeping and aroused brain. Science 1993; 262:679-685 